\title{
An ultrafast and flexible LC-MS/MS system paves the way for machine learning driven sample processing and data evaluation in early drug discovery
}

Tim T. Häbe ${ }^{*}, 1$, Christian Späth ${ }^{2}$, Steffen Schrade ${ }^{1}$, Wolfgang Jörg ${ }^{2}$, Roderich D. Süssmuth ${ }^{3}$, Daniel Bischoff ${ }^{1}$, Andreas H. Luippold ${ }^{*}, 1$

${ }^{1}$ Boehringer Ingelheim Pharma GmbH \& Co. KG, Drug Discovery Sciences, Birkendorfer Strasse 65, 88397 Biberach an der Riss, Germany

2 Boehringer Ingelheim Pharma GmbH \& Co. KG, ISEE Infrastructure, Safety, Environment and Engineering, Birkendorfer Strasse 65, 88397 Biberach an der Riss, Germany

${ }^{3}$ Institut für Chemie, Technische Universität Berlin, Strasse des 17. Juni 124, 10623 Berlin, Germany

* Authors for correspondence:

Tel.: +497351544242

Fax: +497351834242

andreas_harald.luippold@boehringer-ingelheim.com

tim.haebe@boehringer-ingelheim.com 
Rationale: Low speed and flexibility of most LC-MS/MS approaches in early drug discovery delays sample analysis from routine in vivo studies within the same day of measurements. A highthroughput platform for the rapid quantification of drug compounds in various in vivo assays was developed and established in routine bioanalysis.

Methods: Automated selection of an efficient and adequate LC method was realized by autonomous sample qualification for ultrafast batch gradients $(9 \mathrm{~s} / \mathrm{sample})$ or for fast linear gradients $(45 \mathrm{~s} /$ sample $)$ if samples require chromatography. The hardware and software components of our Rapid and Integrated Analysis System (RIAS) were streamlined for increased analytical throughput via state-of-the-art automation while keeping high analytical quality.

Results: Online decision-making was based on a quick assay suitability test (AST) based on a small and dedicated sample set evaluated by two different strategies. $84 \%$ of the acquired data points were within $\pm 30 \%$ accuracy and $93 \%$ of the deviations between the lower limit of quantitation (LLOQ) values were $\leq 2$-fold compared to standard LC-MS/MS systems while speed, flexibility and overall automation was significantly improved.

Conclusions: The developed platform provided an analysis time of only 10 min (batch-mode) and 50 min (gradient-mode) per standard pharmacokinetic (PK) study (62 injections). Automation, data evaluation and results handling were optimized to pave the way for machine learning based decision-making regarding the evaluation strategy of the AST. 


\section{Introduction}

Modern drug discovery processes are based on the close collaboration of various disciplines from the fields of biology, chemistry and medicine. Already early topics like efficacy and toxicology must be addressed in order to diminish potential compound dropouts at later stages and thus the rapid assessment of the compound's pharmacokinetic (PK) and pharmacodynamic (PD) properties is key. Since more than two decades the standard technology to analyze samples from PK and PD studies is liquid chromatography coupled to triple quadrupole mass spectrometers (LC-MS/MS). The progress of mass spectrometry (MS) techniques regarding high-speed approaches for labelfree assays already demonstrated the capabilities and developments towards sub-second sample cycles. RapidFire (RF) systems provide a medium throughput capacity with cycle times of 7-20 s, including a quick solid-phase enrichment/cleanup step $^{1-6}$ or in BLAZE-mode $2.5 \mathrm{~s}$ via direct injection. ${ }^{7}$ Matrix-assisted laser desorption/ionization-time-of-flight (MALDI-TOF) MS with a sample throughput of up to $0.4 \mathrm{~s} / \mathrm{sample}^{8-11}$ and acoustic droplet ejection open port interface (ADE-OPI) MS with up to 6 samples/s are pushing the speed limits even further. ${ }^{12-15}$

Over the years, for the measurement of samples from in vitro studies, the RF-based initial RIAS system proved the suitability for this type of samples ${ }^{5,6}$. Reaching further, we aimed at the transfer of this technical set-up to the measurement of samples from in vivo studies, albeit slower chromatographic steps are often still a required standard procedure. Hence, combining the speed, automation and flexibility of the first RIAS system with the option of liquid chromatography (LC) for samples from specific in vivo assays offers the chance to accelerate the whole in vivo pipeline. However, a fast decision finding is needed to direct samples either to fast detection methods without chromatography, or to the slower path with applied LC.

To cope with the tremendously increasing sample numbers for general LC-MS applications, multiple methodologies around the whole LC-MS pipeline were developed in the last decades. The utilization of pooling methods for samples, ${ }^{16-18}$ UTLC,${ }^{19-21}$ monolithic columns, ${ }^{18,22,23}$ multiplexed approaches ${ }^{23-27}$ and finally faster and more sensitive mass spectrometry systems ${ }^{28,29}$ paved the way for automated high-throughput LC-MS setups.

The goal was to achieve lower sample cycle times and the reduction of human interference with the analytical system while being supported by an automated bioanalytical state-of-the-art workflow management system. This system should support the entire workflow, from the experimental design (e.g. compound selection, sample ID assignment) throughout the in vivo 
experiments (e.g. compound administration and sampling), sample preparation (e.g. dilution factors, calibration and QC samples), analytical processes (e.g. SPE, chromatography, MS parameters), and subsequent data evaluation and determination of PK parameters.

As previously proved, for routine in vitro applications the major key factors, reliability and throughput were already addressed by our initial RIAS platform. ${ }^{5,6}$ This existing platform combined a modified high-throughput liquid handling LC-MS/MS system with a customized master software merging tailor made databases, data processing and reporting options. The herein reported second generation of the RIAS platform (RIAS 2) was developed in order to extend the range of applications to in vivo pharmacokinetic and pharmacodynamics samples. The development was intended to involve chromatography when needed and to solve the next hurdles in lab automation, namely real time monitoring, online decision-making and validation procedures. State-of-the-art bioanalytical systems should take advantage of a well-defined system suitability test (SST) to check the validity of the MS compound tuning process and the entire sample processing procedure in a fully automated way. Various software tools for compound optimization and data processing were reported and contributed to a streamlined process. ${ }^{29-31}$ The RIAS 2 system provides several functionalities: 96-well and 384-well sample plates handling, switching automatically between various ADME assay properties, optional MS compound optimization if required and an automatic so called assay suitability test (AST). This AST is crucial to enable a robust operation of the RIAS system, while minimizing measurement time via fully automated batch/gradient decision. Therefore, a series of test injections was used to determine signal quality in both, batch and gradient mode. Two strategies were developed and investigated to compare a conventional approach and an advanced approach based on various machine learning (ML) tools. ${ }^{33,34}$ 


\section{Experimental}

\subsection{Chemicals and materials}

Acetonitrile (gradient grade), Ammonium acetate (p.a.) and ammonia solution (25\%, p.a.) were purchased from Merck (Darmstadt, Germany), methanol (gradient grade) from J.T. Baker (Deventer, Netherlands), formic acid (FA, p.a.) and trifluoroacetic acid (TFA, reagent grade) from Sigma Aldrich (St. Louis, MO, USA). Purified water was prepared via Elgastat Maxima HPLC (Elga Ltd., High Wycombe Bucks, UK). The $0.8 \mathrm{~mL} 96$ well plates and the "Easy Peel" sealing mats were obtained from Thermo Scientific (Hamburg, Germany). An aqueous mobile phase was prepared of $99.9 \%$ water, $0.09 \%$ FA and $0.01 \%$ TFA and an organic mobile phase of $99.9 \%$ acetonitrile-methanol $(1: 1, V / V), 0.09 \% \mathrm{FA}$ and $0.01 \%$ TFA.

\subsection{Validation experiments}

25 in vivo studies (44 different compounds from Boehringer Ingelheim research projects) were randomly selected from our routine drug discovery process to cover a broad range of analytes and sample matrices. Typical studies were executed based on sampling from plasma, cerebrospinal fluid, brain, and muscle after peroral (p.o.), intravenous (i.v.) or subcutaneous (s.c.) compound administration to rats (Wistar Han and Lister Hooded Crl:LIS) and mice (C57BL/6 and NMRI). For sample preparation, $5 \mu \mathrm{L}$ plasma (blank plasma for quality controls and calibration levels) was transferred to a V-bottomed 96-well plate (AB-1058, Thermo Scientific). An ECHO 550 (Labcyte, San Jose, California) liquid handler was used to spot the respective volume of DMSO stock solutions to obtain typical calibration levels between $0.5 \mathrm{nmol} / \mathrm{L}$ and $20 \mu \mathrm{mol} / \mathrm{L}$ as well as the quality control levels. Protein precipitation was initialized by adding $70 \mu \mathrm{L}$ acetonitrile-methanol (50:50, $V / V)$ via the Microlab STAR liquid handling platform (Hamilton, Bonaduz, Switzerland) in mixing mode. After freezing at $-20^{\circ} \mathrm{C}$ for $10 \mathrm{~min}$ and centrifugation at $\sim 3,200 \times \mathrm{g}$ for $5 \mathrm{~min}$, $30 \mu \mathrm{L}$ of the supernatant was transferred into $270 \mu \mathrm{L}$ aqueous formic acid $(0.1 \%)$ placed in a 96deepwell plate (AB-0859, Thermo Scientific). After shaking for 1 min on a VARIOMAG Monoshake (Thermo Scientific), the plate was centrifuged at $\sim 3,200 \times g$ for 5 min and was ready for check-in at the plate-hotel of the RIAS 2 platform.

\subsection{Conventional LC-MS/MS setup for comparison}

The comparative LC-MS/MS systems consisted of a binary pump (G1312A, Agilent), a CTC HTS PAL autosampler (CTC Analytics AG, Zwingen, Switzerland), and an API 4000 Triple 
Quadrupole mass spectrometer (SCIEX, Darmstadt, Germany) which we used as our "workhorse" analytical system.

Samples for the validation experiments were chromatographed via a 2.0 min linear gradient from $0.1 \%$ aqueous formic acid to $0.1 \%$ formic acid in acetonitrile-methanol $(50: 50, V / V)$ on a Kinetex, $2.6 \mu \mathrm{m}, 30 \times 2.1 \mathrm{~mm}$ C18 column (Phenomenex, Aschaffenburg, Germany).

\subsection{RIAS 2 hardware setup}

The RIAS 2 system (Figure 1) is a further development of our initial RIAS system which was described in detail in previous studies. ${ }^{5,6}$ It is based on the RapidFire ${ }^{\mathrm{TM}}$ system (Agilent, Waldbronn, Germany) equipped usually with C4 solid phase extraction (SPE) cartridges ( $4 \mu \mathrm{L}$ bed volume, G9203A, Agilent) for batch mode. In order to achieve maximum sensitivity a 6500 QTRAP mass spectrometer (SCIEX) was implemented into the system.

To enable the gradient mode, the isocratic pump 3 of the initial RIAS system (Agilent 1200, Agilent, Waldbronn, Germany) was replaced by a low delay volume pump (Agilent 1290). Directly before the MS system ion source, a column selection valve (Cheminert C5-1006D, VICI International, Schenkon, Switzerland) and a Expedite 10x2.1 mm column packed with Alltima $5 \mu \mathrm{m}$ C18 (GRACE, Worms, Germany) was installed (Figure 3d).

\subsection{RIAS 2 hardware operations}

During the whole process of sample sipping, SPE and batch/gradient mode, a constant mobile phase flow to the MS system was provided by pump 3 (red flow, Figure 3). The sample aspiration time was set to $250 \mathrm{~ms}$ to sip the sample into the sampling loop, while the SPE cartridge was equilibrated via aqueous mobile phase from pump 1 at a flow rate of $1.5 \mathrm{~mL} / \mathrm{min}$ (green flow, Figure 3a).

By switching valve 1, the sample portion was transferred to the SPE cartridge by pump 1 via aqueous mobile phase. Due to the high mobile phase flow, this $3000 \mathrm{~ms}$ solid phase extraction step also provided a quick sample clean-up (blue flow, Figure 3b).

For batch mode and gradient mode, the sample loop and capillaries between valve 1 and valve 2 were flushed via pump 2 (orange flow, Figure 3c/d). In batch mode, the analytes were eluted for $3000 \mathrm{~ms}$ with a simple step gradient to $100 \%$ organic mobile phase via pump 3 (Figure 2) from the SPE cartridge. The transfer to the MS system was realized directly via bypass on the column selection valve 5 at a flow rate of $1.25 \mathrm{~mL} / \mathrm{min}$ (Figure $3 \mathrm{c}$ ). Batch mode resulted in a total cycle time of $\sim 9$ s/sample. 
In gradient mode, the analytes were eluted from the SPE cartridge and separated on the C18 column after switching of valve 5 (Figure 3c). Using a $36 \mathrm{~s}$ linear gradient from $95 \%$ aqueous to $100 \%$ organic mobile phase (Figure 2) via pump 3 at a reduced flow rate of $0.6 \mathrm{~mL} / \mathrm{min}$ resulted in a total cycle time of $\sim 45 \mathrm{~s} /$ sample.

\subsection{Software architecture}

The RIAS master software written in LabVIEW v8.6 and later LabVIEW 15 SP1 (National Instruments, Austin, TX) controlled the entire RIAS 2 platform. The modified RapidFire ${ }^{\mathrm{TM}}$ as well as the customized plate handling robot Katana 450 (Neuronics, Zurich, Switzerland) were controlled to pick 96 well or 384 well sample plates from one of the hotels (total capacity: 36 plates). Likewise, the RIAS software controlled the additional gradient pump and the new column selection valve for gradient mode.

A customized version of DiscoveryQuant 2.3 (Sciex) controlled the 6500 QTRAP mass spectrometer (SCIEX). System parameters were optimized for each of the compounds prior to sample analysis in MRM mode with nominal resolution (Q1 and Q3 at 0.7 Da). After successful compound optimization MS parameters DP, CE, EXP, Q1 and Q3 were stored in our central tuning database and can be re-used when the compound enters again the analytical process in another study. Tuning parameters from different MS systems and vendors were adjusted empirically by the in-house software AIMS (Automated In vivo Mass spectrometry Software) to enable a common database and an easy exchange between all instruments and platforms.

According to the "active barcoding" principle ${ }^{5}$ the barcode automatically provides all necessary information for the analytical process. Active barcoding was used for the processing of in vivo samples and facilitated the utilization of the RIAS 2 platform for both, in vivo and in vitro samples either in batch mode or in gradient mode. To enable the data input/output of the complete in vivo sample pipeline (e.g. sample plate layout, sample lists and parameters for MS data acquisition and processing), the master software uses the connection to the AIMS software and its database. To process and evaluate the MS data acquired in the high-throughput (HT) mode ${ }^{32}$ an advanced version of the GMSU ${ }^{\mathrm{TM}}$ software v.8.4.22 (Gubbs Inc., Alpharetta, GA) was used. This software combined sample injection time points (timestamp of valve switching events) with the MS raw data and outputs the signal area values corresponding to the respective sample and assay parameters. 
The RIAS 2 hardware and software was based on in-house customized solutions of commercially available components from different vendors. Nevertheless, the scientific and automation background of the presented investigations can be transferred to any other similar platform.

\subsection{Conventional AST evaluation}

For efficient utilization of the fast batch mode (whenever possible) or gradient mode (as second choice), an assay suitability test (AST) was established. Conventional processing algorithms were applied to evaluate the AST test injection series (Figure 4). Therefore, the area under the curve (AUC) for the main peak of each injection was calculated. Additionally, the AUC of the second largest peak in the "Unknown" sample injection of the gradient mode was calculated to trace potential interferences of metabolites from the compound of interest. Due to the lack of chromatography in the batch mode, the AUC of a metabolite could be erroneously summed up with the main peak and thus alter the respective signal. Subsequently, the resulting quality criteria were evaluated to determine whether to run batch or gradient mode (Table 1). In this approach, an empirical threshold had to be found for each criterion to manage a suitable online decision-making.

\subsection{Online decision-making}

The complete model consists of two signal-processing steps, followed by two machine learning (ML) classifiers (Figure 5). In the first pre-processing step, the signal peaks are excised from the chronogram to extract the sparse information from the complete signal. Subsequently, a set of features was calculated for each injection, including e.g. AUC and maximum peak height. The resulting 10 feature vectors were fed into a Random Forrest Classifier, to obtain an intermediate, 10-dimensional decision vector. ${ }^{33}$ A subsequent Multi-Layer perceptron makes the final decision by mapping this vector to one of the three above-mentioned categories. ${ }^{34}$ 


\section{Results and Discussion}

\subsection{Strategy for RIAS 2}

The RIAS 2 platform was introduced as an further development of the initial RIAS platform ${ }^{6}$ to meet all demands regarding in vivo sample processing in a streamlined process. The extension from previously demonstrated applications of ADME assays (e.g. metabolic stability, CaCo-2 or PAMPA permeability and CYP450 inhibition) including assay type recognition and MS compound optimization with the initial RIAS platform under real-life conditions, ${ }^{5}$ to in vivo assays was an important step to take for routine high-throughput bioanalysis. Challenges were the sample data input, access to a centralized MS tuning database and the optimization of the whole hardware setup. This comprised the expansion to sensitive, selective and fully automated MS data acquisition with ML decision-making for the most efficient sample analysis strategy.

The two investigated decision-making processes, if a sample can be analyzed in the $9 \mathrm{~s}$ batch mode, the $45 \mathrm{~s}$ gradient mode or has to be rejected by RIAS 2 and then is transferred to conventional LCMS/MS mode, was the most critical step to achieve overall fast cycle times for all samples in the analytical pipeline. Thus, a robust and fast decision-finding has been investigated regarding two different approaches.

\subsection{Platform development}

The RIAS 2 platform and its RIAS master software were designed to communicate according to handshake principles. Including the plate handling robot, the LC column selection valve, and the 6500 QTRAP mass spectrometer (Sciex), all hardware was precisely timed and triggered according to the sample and assay demands. This data input was realized by the connection to the PIDS (in-house in vivo laboratory information management system, LIMS) and AIMS software instances.

Fast in vivo bioanalysis requires an efficient process, including a reliable data handling process: Active barcoding triggers not only the hardware settings, but in regard of the different assay demands, also the layout of data input and output for MS data evaluation. Thus, a clear distinction between tuning and different sample plates just randomly put in the plate hotel after check-in via barcode scan was most important to guarantee a stable, unattended operation for up to 36 plates. The clear decision if compound tuning parameters were already available in the database or a complete compound tuning was necessary was one critical aspect in order to accelerate this procedure. $^{6}$ 
The RIAS software combined all involved software and database instances in an automated and user-friendly process, from assay layout and data acquisition parameters to MS data processing and evaluation with GMSU (Gubbs Inc.). Moreover, the compound optimization (tuning) or sample measurement mode as well as sequences of multiple sample plates and assays can be submitted and controlled via remote access. The unattended operation and the general suitability and flexibility for various assays and compounds was most important to meet the demands of a high-throughput environment.

For the conventional AST, samples were grouped into PK studies and PD studies. For PK studies the aspired LLOQ threshold of a compound was defined to be $\leq 10 \mathrm{nmol} / \mathrm{L}$ to cover the terminal phase. Additionally, for PD studies, a sample of the minimal dose of compound administration was tested to confirm sufficient sensitivity of the method. Information about the respective studies was sourced from AIMS, our central database gathering all workflow information (e.g. dose of compound administration, sampling timepoints, dilution factors, calibration levels, and MS parameters). Additional criteria on ion suppression were determined: the signal area of the internal standard in the "unknown" sample must be between $50 \%$ and $150 \%$ of its signal area in a representative calibration level (C06 ref, Figure 4). To confirm a suitable carry-over state of the system, this value was also recognized for each blank sample.

\subsection{Online decision-making via AST}

The most critical time aspect was the decision whether a sample could be processed in batch mode, gradient mode or had to be rejected to be measured by conventional LC-MS/MS. To avoid the cumbersome and time-consuming tuning process of the conventional AST evaluation, a new approach was developed by utilizing machine learning (ML). This approach combines the knowledge of the expected measurement signals, used in the pre-processing and feature extraction, with the flexibility of ML. The ML approach was trained on an existing, labelled database comprising 1873 results of individual in vivo samples. From this set the machine-learning approach assigned the samples to batch mode (51\%), to gradient mode (34\%), and to conventional LCMS/MS (none, 15\%). This sample set was split randomly in a ML-typical ratio of 80/20 ratio: 1500 samples were subjected to the ML training and 373 samples to the validation. A classifier was used to sort the acquired AST series (Figure 4) into one of the three different categories: batch, gradient or none. This fully automated batch/gradient decision was established to implement a robust operation of the RIAS system. Subsequently, signal quality of only 10 test injections was required 
for decision-making to route the sample analysis strategy to batch mode, gradient mode or to send the respective sample to conventional LC-MS/MS. Thus, the overall measurement time was minimized at still highest data quality. The training results based on the 1500 pre-labelled samples are indicated by the confusion matrix (Figure 5).

Due to the utilization of this prior knowledge-based ML AST (training based on the labelled database), this comparatively small number of samples $(n=1500)$ is sufficient to achieve an accuracy of $98 \%$ in the prediction of batch measurements and $96 \%$ in the prediction of gradient measurements. The conventional AST strategy was compared with the ML AST strategy. This revealed that $9 \%$ of samples rejected to conventional LC-MS/MS by the conventional AST were classified correctly for gradient mode by the ML AST. However, manual inspection of these differently labelled ASTs showed that $>60 \%$ of the samples could be measured in the faster batch mode and were mislabeled by the conventional algorithm. This demonstrated that the ML based approach could model the conventional approach and even improve its performance.

\subsection{Batch mode versus gradient mode}

To enable the gradient mode, the isocratic pump of the initial version of the RIAS platform was replaced by a gradient pump and a column selection valve expanded the flexibility to select one of up to five columns between the SPE cartridge and the MS system (Figure 3d). The sixth valve channel was used as bypass for the batch mode, where all compounds trapped on the SPE cartridge were eluted simultaneously into the MS system, resulting in one injection peak for each sample (Figure 3c). In the gradient mode, interfering components were separated by chromatography on the additional chromatography column resulting in discrete peak shapes for different components of one sample. The batch mode with a cycle time of $9 \mathrm{~s}$ was clearly preferred whenever possible, compared to the $45 \mathrm{~s}$ gradient mode.

For example, a typical p.o. PK study consists of approximately 60 injections (i.e. unknown samples from three animals at six timepoints, including quality control samples, calibration samples, and blanks) and can be analyzed within $10 \mathrm{~min}$ (batch mode) compared to $50 \mathrm{~min}$ if fully measured in gradient mode.

\subsection{Comparison of the RIAS 2 setup with conventional LC-MS/MS}

A dataset with 944 data points from various in vivo studies was used to compare RIAS 2 and conventional LC-MS/MS (Figure 7). From this dataset, 894 data points (94.7\%) were successfully generated with the RIAS 2 system. The remaining 5.3\% data point are linked to samples with $c(t)$ - 
values of terminal phases of pharmacokinetic (PK) studies. This is actually the case when the actual systemic concentration of the compound of interest in the animal is very low. The correlation between both methods revealed a $R^{2}$ of 0.9863 (Figure 7a) and a Bland-Altman plot visualized the 797 datapoints (84.4\%) within a $\pm 30 \%$ inaccuracy limit, 70 datapoints $(7.4 \%)$ within a $\pm 50 \%$, and 27 datapoints (2.9\%) within a $\pm 100 \%$ (Figure $7 b$ ). This performance in combination with the fast and flexible RIAS 2 pipeline demonstrated the suitability for routine bioanalytical tasks. 


\section{Conclusions}

In terms of sensitivity and selectivity compared to nowadays standard LC-MS/MS setups, the RIAS 2 system combines linearity for a wide concentration range (typically $>1 \mathrm{nM}$ to $<10 \mu \mathrm{M}$ ) of complex in vivo samples where selectivity is most important. The standardization of workflows for in vivo samples is a key benefit while maintaining flexibility which is required for an agile response on various analytical tasks.

The AIMS (automated in vivo mass spectrometry software) package enables not only an increased process stability and high throughput, but also is the base for automated decision-finding to choose the most efficient setup of analysis. The demonstrated machine learning techniques for interpretation of the AST was only the first step in the direction of an improved data handling and feedback loops to further improve the complete RIAS 2 workflows from sampling to data interpretation and reporting. Using the current GMSU HT-mode, data processing was already eased and accelerated significantly.

We demonstrated that a ML approach can be used, to decide the measurement strategy for the new RIAS 2 system. This allowed to minimize measurement time, while keeping data quality high and at the same time to sort out samples, which must be measured in a conventional LC-MS/MS system. The proposed ML model avoided the cumbersome tuning of AST evaluation parameters, which allows easy and fast adaption of the system to new requirements imposed by new compounds. This enormously reduced the time- and cost-intense setup of the system.

At a high level of automation, the rapid sample analysis of $9 \mathrm{~s}$ for batch mode and $45 \mathrm{~s}$ for gradient mode outperforms conventional LC-MS/MS and other ESI-based techniques with integrated SPE or chromatography. The RIAS 2 platform with its "active barcoding" sample plate check-in addresses the need for speed in early research projects and therefore significantly contributes to an advanced strategy for in vitro and in vivo samples.

The platform allows "fast-track" bioanalysis, which is of particular interest in the drug discovery process especially in pharmacodynamic (PD) experiments where multiple doses are applied. Assuming sample preparation, data handling and processing are adapted to the speed of the analytical system, the results from the first dosing event are available before the next dosing interval. This enables monitoring whether the active principle of the administered compound meets the predicted concentration profile and facilitates a dose adjustment if needed. 


\section{Acknowledgement}

We greatly acknowledge Andreas Barthold, Chris Cantow and the entire Research Bioanalysis Team for their outstanding skills in tuning, running the system, pushing the limits, and of course performing all the study measurements. But most importantly for their empowerment to strive for the next level of automation and efficiency while keeping high data quality in modern Bioanalysis. We also gratefully acknowledge the support from our ISEE colleagues of Boehringer Ingelheim, namely Max Link and Michael Haas. 


\section{References}

1. Sawyer WS, Srikumar N, Carver J, et al. High-throughput antibody screening from complex matrices using intact protein electrospray mass spectrometry. Proc Natl Acad Sci US A. 2020;117(18):9851-9856.

2. Veach BT, Mudalige TK, Barnes PJ, Baker CA. Quantitative Screening Method for Erythromycin and Tylosin in Honey Using RapidFire Mass Spectrometry. J AOAC Int. 2019;102(1):297-301.

3. Hutchinson SE, Leveridge MV, Heathcote ML, et al. Enabling lead discovery for histone lysine demethylases by high-throughput RapidFire mass spectrometry. J Biomol Screen. 2012;17(1):39-48.

4. Wu X, Wang J, Tan L, et al. In vitro ADME profiling using high-throughput RapidFire mass spectrometry: cytochrome p450 inhibition and metabolic stability assays. J Biomol Screen. 2012;17(6):761-772.

5. Luippold AH, Arnhold T, Jörg W, Krüger B, Süssmuth RD. Application of a rapid and integrated analysis system (RIAS) as a high-throughput processing tool for in vitro ADME samples by liquid chromatography/tandem mass spectrometry. $J$ Biomol Screen. 2011;16(3):370-377.

6. Luippold AH, Arnhold T, Jörg W, Süssmuth RD. An integrated platform for fully automated high-throughput LC-MS/MS analysis of in vitro metabolic stability assay samples. Int J Mass Spectrom. 2010;296(1-3):1-9.

7. Bretschneider T, Ozbal C, Holstein M, et al. RapidFire BLAZE-mode is boosting ESI-MS toward high-throughput-screening. SLAS Technol. 2019;24(4):386-393.

8. Simon RP, Winter M, Kleiner C, et al. MALDI-TOF-Based Affinity Selection Mass Spectrometry for Automated Screening of Protein-Ligand Interactions at High Throughput. SLAS Discov. 2020;26(1):44-57.

9. Simon RP, Winter M, Kleiner C, et al. MALDI-TOF mass spectrometry-based highthroughput screening for inhibitors of the cytosolic DNA sensor cGAS. SLAS Discov. 2020;24(4):372-383. 
10. Winter M, Ries R, Kleiner C, et al. Automated MALDI target preparation concept: Providing ultra-high-throughput mass spectrometry-based screening for drug discovery. SLAS Technol. 2017;2(24):209-221.

11. Haslam C, Hellicar J, Dunn A, et al. The evolution of MALDI-TOF mass spectrometry toward ultra-high-throughput screening: 1536-well format and beyond. $J$ Biomol Screen. 2016;21(2):176-186.

12. Zhang J, Zhang Y, Liu C, et al. Acoustic ejection/full-scan mass spectrometry analysis for high-throughput compound quality control. SLAS Technol. 2020;https://doi.org/10.1177/2472630320967625.

13. Häbe TT, Liu C, Covey TR, et al. Ultrahigh-throughput ESI-MS: sampling pushed to six samples per second by acoustic ejection mass spectrometry. Anal Chem. 2020;92(18):1224212249.

14. DiRico KJ, Hua W, Liu C, et al. Ultra-high-throughput acoustic droplet ejection-open port interface-mass spectrometry for parallel medicinal chemistry. ACS Med Chem Lett. 2020;11(6):1101-1110.

15. Liu C, van Berkel GJ, Cox DM, Covey TR. Operational modes and speed considerations of an acoustic droplet dispenser for mass spectrometry. Anal Chem. 2020;92(24):15818-15826.

16. Tolonen A, Petsalo A, Turpeinen M, Uusitalo J, Pelkonen O. In vitro interaction cocktail assay for nine major cytochrome P450 enzymes with 13 probe reactions and a single LC/MSMS run: analytical validation and testing with monoclonal anti-CYP antibodies. J Mass Spectrom. 2007;42(7):960-966.

17. Kim M-J, Kim H, Cha I-J, et al. High-throughput screening of inhibitory potential of nine cytochrome P450 enzymes in vitro using liquid chromatography/tandem mass spectrometry. Rapid Commun Mass Spectrom. 2005;19(18):2651-2658.

18. Peng SX, Barbone AG, Ritchie DM. High-throughput cytochrome p450 inhibition assays by ultrafast gradient liquid chromatography with tandem mass spectrometry using monolithic columns. Rapid Commun Mass Spectrom. 2003;17(6):509-518. 
19. Xu R, Manuel M, Cramlett J, Kassel DB. A high throughput metabolic stability screening workflow with automated assessment of data quality in pharmaceutical industry. $J$ Chromatogr A. 2010;1217(10):1616-1625.

20. Bateman KP, Kellmann M, Muenster H, Papp R, Taylor L. Quantitative-qualitative data acquisition using a benchtop orbitrap mass spectrometer. J. Am. Soc. Mass Spectrom. 2009;20(8):1441-1450.

21. Plumb RS, Potts WB, Rainville PD, et al. Addressing the analytical throughput challenges in ADME screening using rapid ultra-performance liquid chromatography/tandem mass spectrometry methodologies. Rapid Commun Mass Spectrom. 2008;22(14):2139-2152.

22. Di L, Kerns EH, Hong Y, Kleintop TA, McConnell OJ, Huryn DM. Optimization of a higher throughput microsomal stability screening assay for profiling drug discovery candidates. $J$ Biomol Screen. 2003;8(4):453-462.

23. Deng Y, Wu J-T, Lloyd TL, Chi CL, Olah TV, Unger SE. High-speed gradient parallel liquid chromatography/tandem mass spectrometry with fully automated sample preparation for bioanalysis: 30 seconds per sample from plasma. Rapid Commun Mass Spectrom. 2002;16(11):1116-1123.

24. Kassel DB. Applications of high-throughput ADME in drug discovery. Curr Opin Chem Biol. 2004;8(3):339-345.

25. Heinig K, Bucheli F. Ultra-fast quantitative bioanalysis of a pharmaceutical compound using liquid chromatography-tandem mass spectrometry. J Chromatogr B. 2003;795(2):337-346.

26. King RC, Miller-Stein C, Magiera DJ, Brann J. Description and validation of a staggered parallel high performance liquid chromatography system for good laboratory practice level quantitative analysis by liquid chromatography/tandem mass spectrometry. Rapid Commun Mass Spectrom. 2002;16(1):43-52.

27. Janiszewski JS, Rogers KJ, Whalen KM, et al. A high-capacity LC/MS system for the bioanalysis of samples generated from plate-based metabolic screening. Anal Chem. 2001;73(7):1495-1501.

28. Yu S, Crawford E, Tice J, Musselman B, Wu J-T. Bioanalysis without sample cleanup or chromatography: the evaluation and initial implementation of direct analysis in real time 
ionization mass spectrometry for the quantification of drugs in biological matrixes. Anal Chem. 2009;81(1):193-202.

29. Rathore R, Corr JJ, Lebre DT, Seibel WL, Greis KD. Extending matrix-assisted laser desorption/ionization triple quadrupole mass spectrometry enzyme screening assays to targets with small molecule substrates. Rapid Commun Mass Spectrom. 2009;23(20):3293-3300.

30. Kieltyka K, Zhang J, Li S, et al. A high-throughput bioanalytical platform using automated infusion for tandem mass spectrometric method optimization and its application in a metabolic stability screen. Rapid Commun Mass Spectrom. 2009;23(11):1579-1591.

31. Smalley J, Xin B, Olah TV. Increasing high-throughput discovery bioanalysis using automated selected reaction monitoring compound optimization, ultra-high-pressure liquid chromatography, and single-step sample preparation workflows. Rapid Commun Mass Spectrom. 2009;23(21):3457-3464.

32. Janiszewski JS, Rogers KJ, Whalen KM, et al. A High-capacity LC/MS system for the bioanalysis of samples generated from plate-based metabolic screening. Anal. Chem. 2001;73(7):1495-1501.

33. Breiman L. Random forests. Mach Learn. 2001;45(1):5-32.

34. Hornik K, Stinchcombe M, White H. Multilayer feedforward networks are universal approximators. Neu Net. 1989;2(5):359-366. 
Table 1. The quality criteria of the conventional AST evaluation to decide batch mode versus gradient mode for the respective compound (CMPD).

\begin{tabular}{|c|c|c|}
\hline \multirow{5}{*}{ 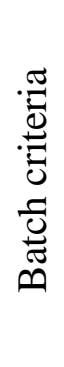 } & Lower level of quantification (LLOQ): & $\frac{C M P D(\text { Blank } 1)}{C M P D(\text { C06ref })}$ \\
\hline & \multirow{2}{*}{ Internal standard suppression (IS $\mathrm{SUP}$ ): } & $I S($ C06ref $)$ \\
\hline & & $\overline{I S(\text { Unknown })}$ \\
\hline & \multirow{2}{*}{ Signal to noise ratio (SNR): } & CMPD $($ Unknown $)$ \\
\hline & & $\overline{C M P D(B \operatorname{lank} 1)}$ \\
\hline \multirow{6}{*}{ 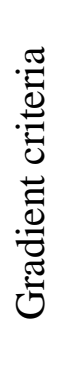 } & \multirow{2}{*}{ Lower level of quantification (LLOQ): } & $C M P D($ Blank 1$)$ \\
\hline & & $\overline{C M P D}($ C06 ref $)$ \\
\hline & \multirow{2}{*}{ Internal standard suppression (IS $\mathrm{SUP}$ ): } & $I S(C 06$ ref $)$ \\
\hline & & $\overline{\text { IS(Unknown })}$ \\
\hline & \multirow{2}{*}{ Side-peak area: } & CMPD $\left(\right.$ Unknown $\left._{\text {main }}\right)$ \\
\hline & & $C M P D\left(\right.$ Unknown $\left._{\text {side }}\right)$ \\
\hline
\end{tabular}




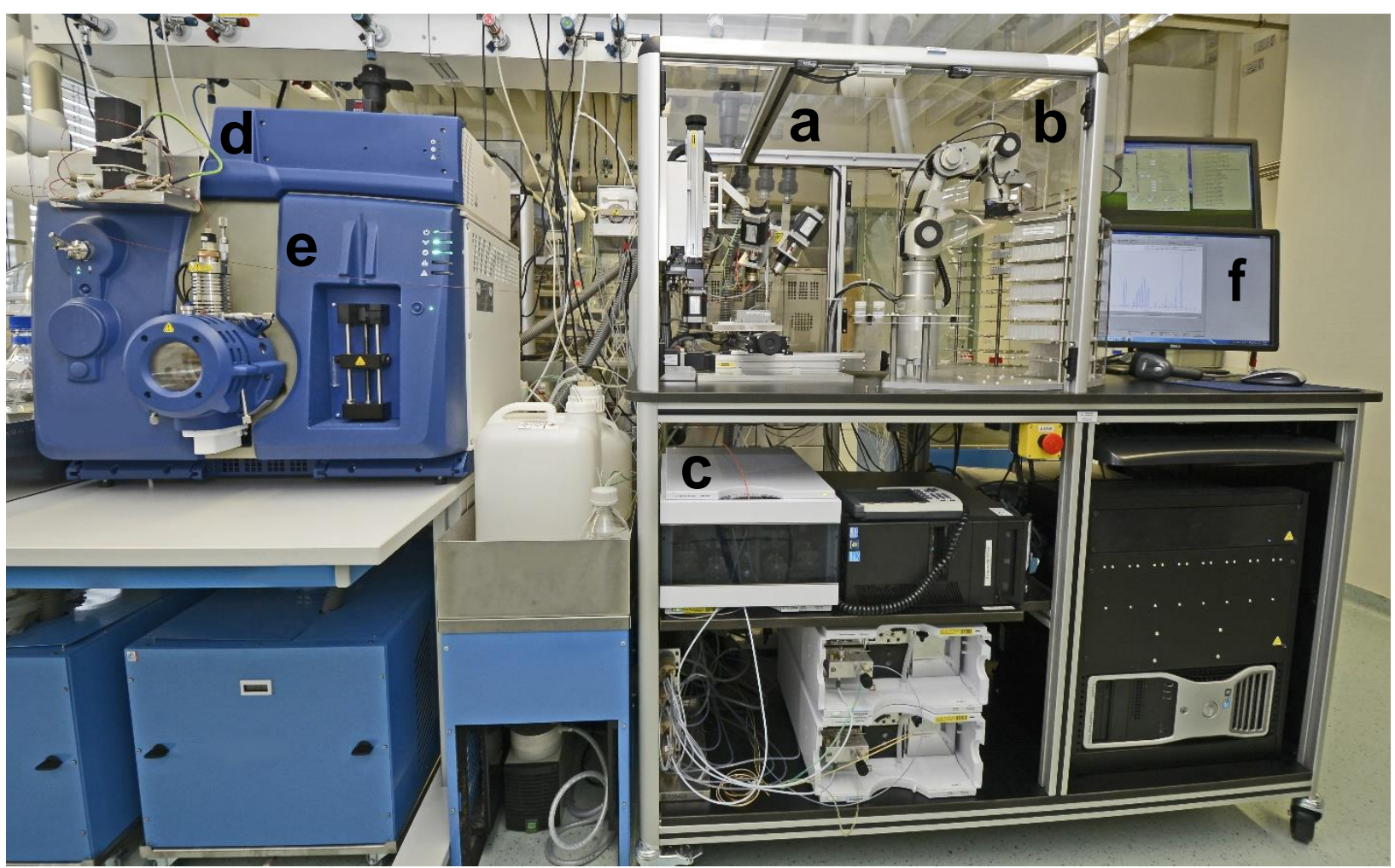

Figure 1. RIAS 2 components: Rapid-Fire ${ }^{\mathrm{TM}}$ injection system (a) with additional plate hotel for up to 36 sample plates (b) and optional gradient option (c) with HPLC column and selection valve (d) for improved selectivity. The state-of-the art mass spectrometer (e) for improved sensitivity and the RIAS master software with additional data processing options for high quality of data handling and processing (f). 


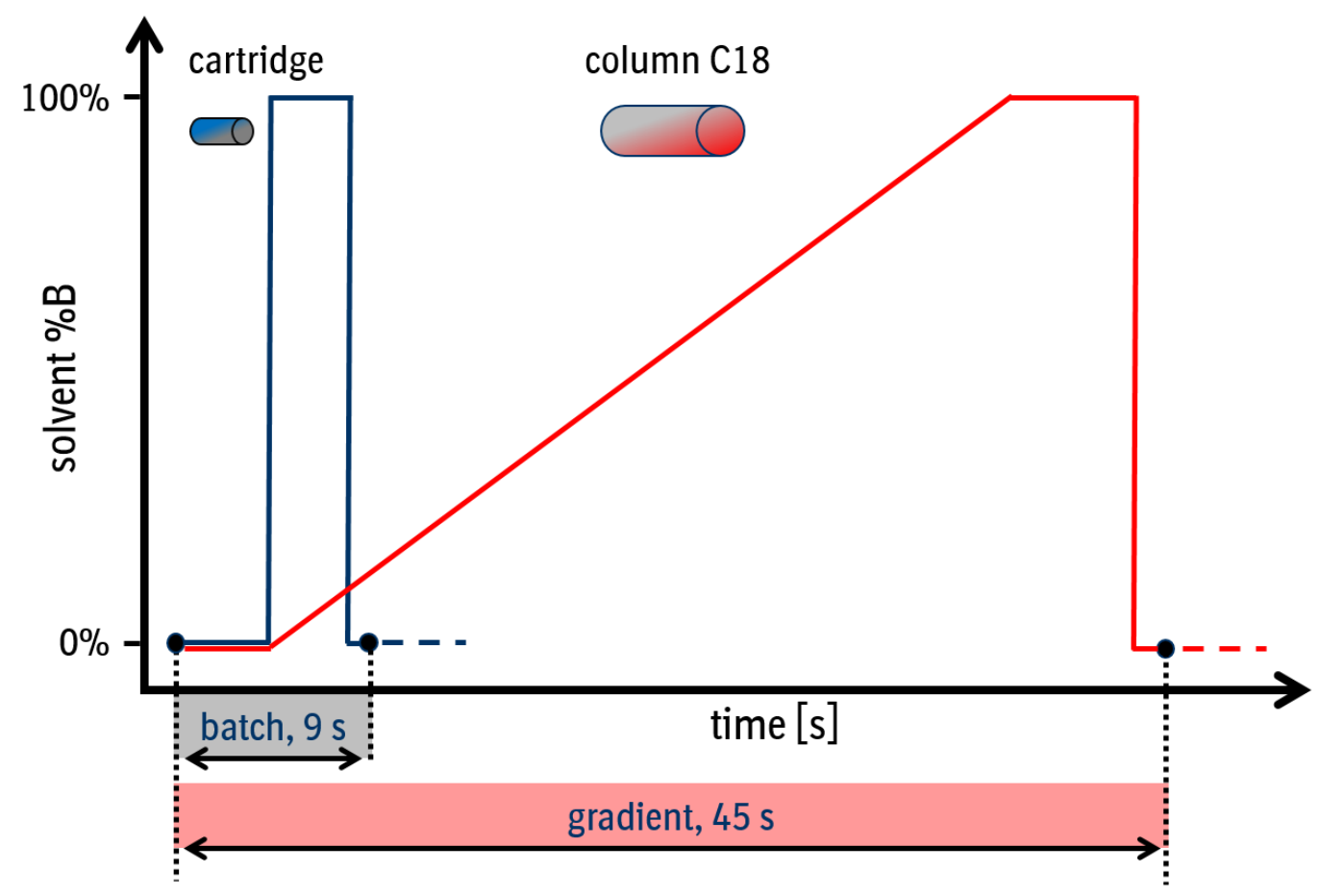

Figure 2. Batch versus gradient mode: The direct shift from solvent $A$ to solvent $B$ during batch mode using the cartridge (blue) and the gradient shift for separation on the C18 column (red). 


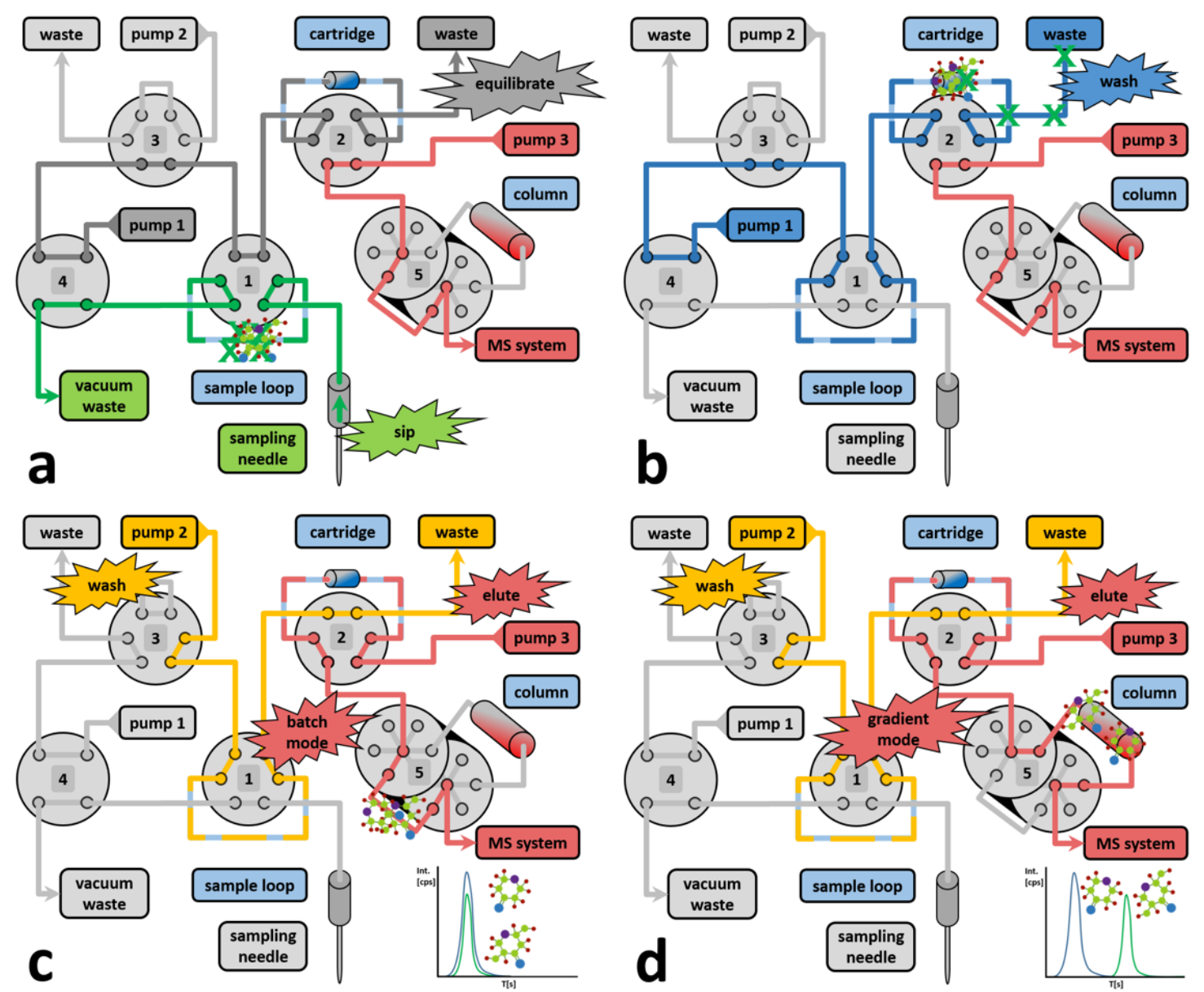

Figure 3. Illustration of the RIAS 2 sampling, compound trapping and the switching between batch mode and gradient mode. The sample is sipped into the sample loop (on valve 1) via vacuum, while the SPE cartridge (on valve 2) is equilibrated via pump 1 (a). The aqueous solvent flow from pump 1 transfers the sample to the SPE cartridge and eliminates matrix components (green $\mathrm{X}$ symbol) not trapped by the cartridge (b). Subsequently, the organic solvent from pump 3 elutes the trapped compounds towards the MS system while the sample loop and tubing are rinsed via pump 2 (c, d). In batch mode, the isocratic solvent flow from pump 3 just passes the column selection valve 5 via bypass directly to the MS system, resulting in one single peak (c). In gradient mode, valve 5 is switched to the LC column to perform chromatography before the separated compounds enter the MS system (d). 


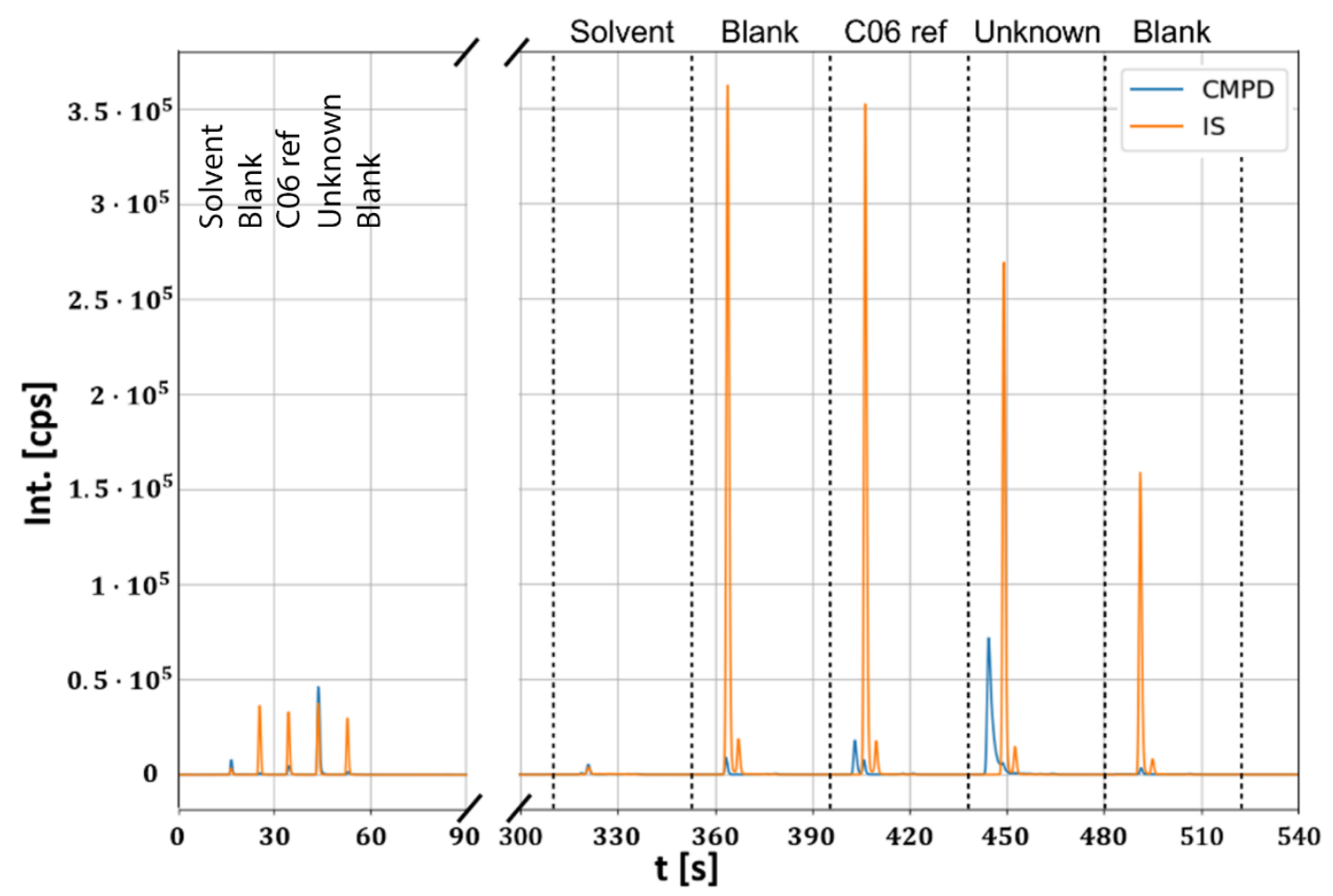

Figure 4. Exemplary assay suitability test (AST) injection series in batch mode $(0-90 \mathrm{~s})$ and gradient mode (300-540 s) for the compound (CMPD, blue) and internal standard (IS, orange). Each test series was based on five injections: solvent, blank, C06 reference, unknown sample and a final blank to check carry over. After batch mode, the column selection valve was switched to gradient mode and the same wells were injected again for chromatography on the analytical column. 


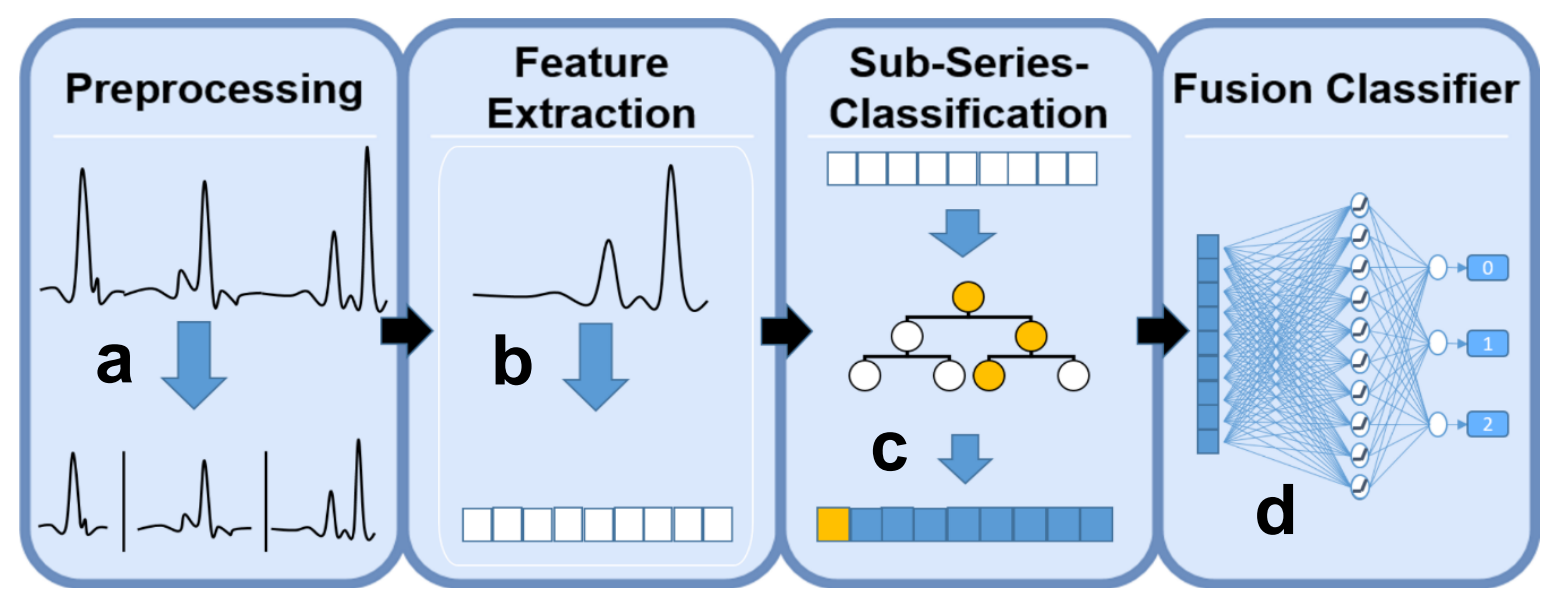

Figure 5. Machine Learning (ML) model for AST evaluation: preprocessing stage for injection peak extraction to densify the sparse information and to reduce the noise level (a). Feature extraction by conventional signal processing (b). Resulting feature vectors were fed into a random forest classifier producing one decision for each feature vector (c). After combination to a 10dimensonal decision vector, the final decision-making stage was based on a fully connected, multilayer, neural network to map decision vectors to one of the three output classes (d). The two ML stages (c) and (d) where trained together on an existing, labelled set of AST data $(n=1500)$. 


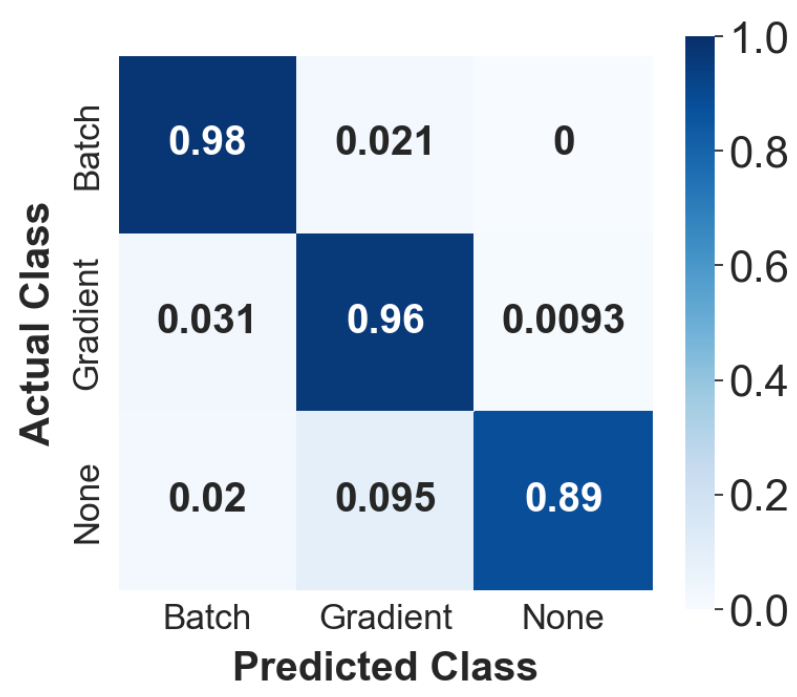

Figure 6. Confusion matrix for the ML based class prediction revealed the suitability for routine analysis. The matrix compares the prediction of the model, trained on 1500 pre-labeled samples with the prediction of the conventional algorithm. An accuracy of $98 \%$ in the prediction of batch measurements and $96 \%$ in the prediction of gradient measurements were achieved, while $9 \%$ of the conventionally None labelled samples were classified as Gradient. 

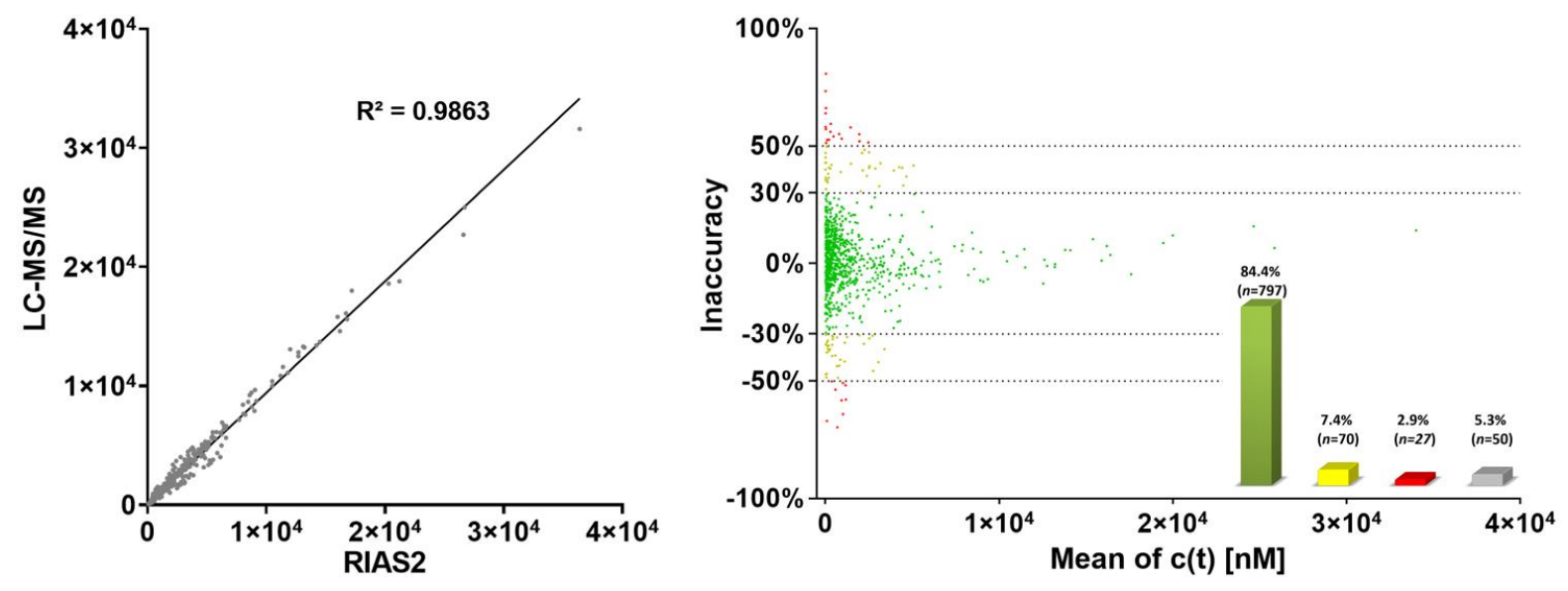

Figure 7. From an exemplary dataset (944 data points), we achieved reasonable peak signals from 894 data points $(94.7 \%)$, while $5.3 \%$ of the datapoints were rejected. All of these $50 \mathrm{c}(\mathrm{t})$-values were linked to the terminal phase of PK studies with lowest $\mathrm{c}(\mathrm{t})$-values. Comparison of conventional LC-MS/MS to RIAS 2 in batch mode $(n=894)$ showing $R^{2}$ of $0.9863(\mathrm{~A})$. The qualification limit of $\pm 30 \%$ was reached for $84.4 \%$ of data points, while $7.4 \%$ reached $\pm 50 \%$ and $2.9 \%$ were still below $\pm 100 \%$. 